\title{
Improvement Construction for Planar G2 Transition Curve Between Two Separated Circles
}

\author{
Zhong $\mathrm{Li}^{1,2}$, Lizhuang $\mathrm{Ma}^{2}$, Mingxi Zhao ${ }^{2}$, and Zhihong $\mathrm{Mao}^{2}$ \\ ${ }^{1}$ Department of Mathematics and Science, Zhejiang Sci-Tech University, \\ Hangzhou, 310018, China \\ lizhongzju@hotmail.com \\ ${ }^{2}$ Department of Computer Science and Engineering, Shanghai Jiao Tong University, \\ Shanghai, 200030, China \\ ma-lz@cs.sjtu.edu.cn, \{Zhaomx, mzh_yu\}@sjtu.edu.cn
}

\begin{abstract}
In this paper, we use the undetermined coefficient method to find a desirable pair of cubic Bezier spirals and a desirable pair of quintic PH spirals to generate planar G2 transition curve between two separated circles. The G2 transition curve can be gotten by the rooting formula, which simplifies the computation, and the ratio of two radii has no restriction, which extends the application area.
\end{abstract}

\section{Introduction}

Transition curves are useful for several Computer Graphics and CAD applications. They may be used for blending in the plane, i.e. to round corners, or for smooth transition between two curves, usually two circular arcs. For some applications, such as the design of highways, railways or robot trajectories, it is desirable that the curvature varies monotonically with arc-length. So the research on G2 transition curve is important [1-3].

There have been some studies for the G2 transition curve when two circles have different positions. The Clothoid or Cornu spiral has traditionally been used in highway design for many years $[2,3]$, but it can not be represented exactly as a NURBS curve, so it is not used easily in standard graphics packages. For two tangent circles, Li and Meek [9] gave the smooth method of arc spline by a pair of rational quadratic Bezier curves or one cubic Bezier curve. For two separated circles, Walton and Meek $[4,6]$ found a family of cubic Bezier spirals and used one of them to construct the transition curve, but it requires the numerical method to solve, and in order to make sure there is one solution, the ratio of two radii sometimes has some restriction. Walton and Meek [5,7] also found a family of quintic $\mathrm{PH}$ spirals and used one of them to construct the transition curve, but it needs the numerical computation, and the ratio of two radii has some limitation too.

In this paper, we give the improvement construction for G2 transition curve between two separated circles. Based on the undetermined coefficient method, we find a pair of cubic Bezier spirals and a pair of quintic $\mathrm{PH}$ curve spirals to generate G2 transition curve. The advantage of improvement method is that 
the transition curve can be gotten by the rooting formula, which simplifies the computation. And the ratio of two radii has no limitation, which extends the application area. The improvement construction of G2 transition curve is more convenient for applications in the fields of CAD and Computer Graphics.

\section{G2 Transition Curve by a Pair of Cubic Bezier Spirals}

The standard cubic Bezier curve can be written as

$$
B(t)=(1-t)^{3} B_{0}+3 t(1-t)^{2} B_{1}+3 t^{2}(1-t) B_{2}+t^{3} B_{3}, t \in[0,1],
$$

where $B_{0}, B_{1}, B_{2}, B_{3}$ are control points of the cubic Bezier curve.

Denote $h_{0}=\left\|B_{1}-B_{0}\right\|, h_{1}=\left\|B_{2}-B_{1}\right\|, h_{2}=\left\|B_{3}-B_{2}\right\|, \theta$ is the angle from $B_{0} B_{1}$ to $B_{1} B_{2}, \phi$ is the angle form $B_{1} B_{2}$ to $B_{2} B_{3}$. From $\mathrm{R}[8]$, we know when $\theta=$ $0, h_{1}=h_{0}$, and $h_{2} \leq \frac{6}{5} h_{1} \cos \phi$, the cubic Bezier curve is a spiral, the curvature at one endpoint is zero, the curvature at another endpoint is a constant, the curvature is monotone for $t \in[0,1]$. Here, we denote $h_{2}=\frac{6}{5} x h_{1} \cos \phi$, where $x$ is a real number and $0<x \leq 1$. Helped by the undetermined coefficient method to find a desirable $x$, we can use the quadratic equation to get a pair of Bezier spirals for planar G2 transition curve in $\mathrm{S}$ shape between two separated circles.

In this case, assume that two circles $\Omega_{0}, \Omega_{1}$ centred at $C_{0}, C_{1}$ with radius $r_{0}, r_{1}, r_{0} \geq r_{1}$, see Fig 1 . We use a pair of Bezier spirals $B_{0}(t), B_{1}(t)$ to construct the G2 transition curve. Suppose $B_{0}(0), B_{1}(0)$ are the joint of two spirals at $B(0)$, points $B_{0}(1), B_{1}(1)$ connect two circles, $T, N$ are the unit tangent vector and the unit normal vector at the joint of two spirals, $T_{a}, T_{b}$ are the unit tangent vector at another endpoints of two curves, the angle from $T$ to $T_{b}$ and the angle from $T_{a}$ to $T$ are both $\phi$.

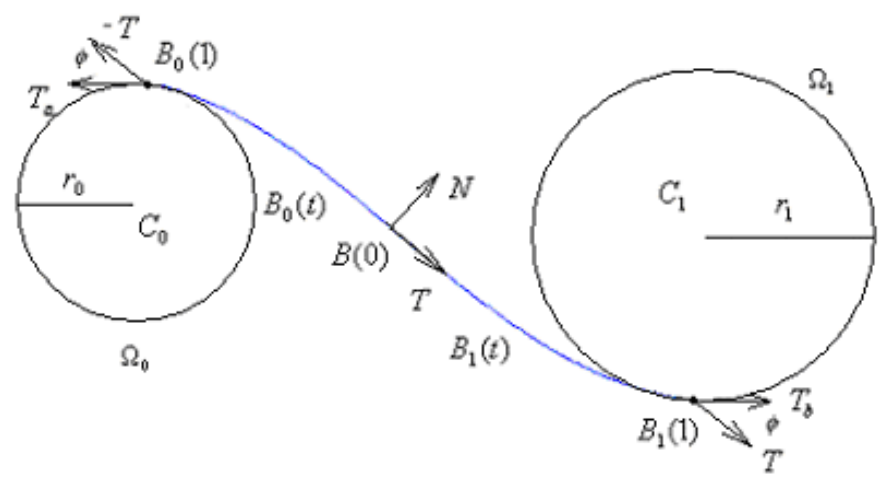

Fig. 1. G2 transition curve in S shape between two separated circles

Let $x=\frac{5}{9}$ and use the geometry relation, we can obtain a quadratic equation

$$
q(\phi)=-\left\|C_{1}-C_{0}\right\| 2 \cos \phi^{2}-8\left(r_{1}+r_{0}\right)^{2} \cos \phi+9\left(r_{1}+r_{0}\right)^{2} .
$$


We can guarantee there is the unique root of $q(\phi)=0$ when $\phi \in\left(0, \frac{\pi}{2}\right)$. This root can be obtained by the rooting formula of quadratic equation. At the same time, since $x=\frac{5}{9}<1$, the cubic Bezier curve is a spiral. And in the computation process, we find the ratio of two radii has no restriction.

Similarly, we can use a pair of cubic Bezier spirals to construct the G2 transition curve in $\mathrm{C}$ shape between two separated circles. The solution can also be gotten by the rooting formula of quadratic equation and the ratio of two radii has no restriction.

\section{G2 Transition Curve by a Pair of Quintic PH Spirals}

Consider the curve $Q(t)=x(t) T_{0}+y(t) N_{0}$ and its derivative with respect to $t$, where $x(t)$ and $y(t)$ are polynomials in $t, T_{0}$ and $N_{0}$ are two orthonormal vectors. The curve $Q(t)$ is said to be a PH curve if $\left\{x^{\prime}(t)\right\}^{2}+\left\{y^{\prime}(t)\right\}^{2}$ can be expressed as the square of a polynomial in $t$. To ensure $Q(t)$ is indeed a $\mathrm{PH}$ curve, define $x^{\prime}(t)$ and $y^{\prime}(t)$ as $x^{\prime}(t)=U^{2}(t)-V^{2}(t)$ and $y^{\prime}(t)=2 U(t) V(t)$, where $U(t) V(t)$ are polynomials. A PH quintic Bezier form of $Q(t)$ is obtained by defining $U(t)$ and $V(t)$ as $U(t)=i(1-t)^{2}+2 j t(1-t)+k t^{2}, V(t)=u(1-t)^{2}+2 v t(1-t)+w t^{2}$.

From $\mathrm{R}[8]$, we know when $u=v=0, i=j$, and $i \geq \frac{7 k}{4(1+\cos \theta)}$, where $\theta$ is the angle between tangents ate two endpoints, the quintic $\mathrm{PH}$ curve is a spiral. Here, we denote $i=j=\frac{7 x k}{4(1+\cos \theta)}, k=\frac{y}{2} \sqrt{7 r \sin \theta}, u=v=0, w=k \sqrt{\frac{1-\cos \theta}{1+\cos \theta}}$, where $x, y$ are real numbers, $x \geq 1, y>0$. Based on the undetermined coefficient method to find the desirable $x, y$, we can use the rooting formula to construct the G2 transition curve in $\mathrm{C}$ shape between two separated circles.
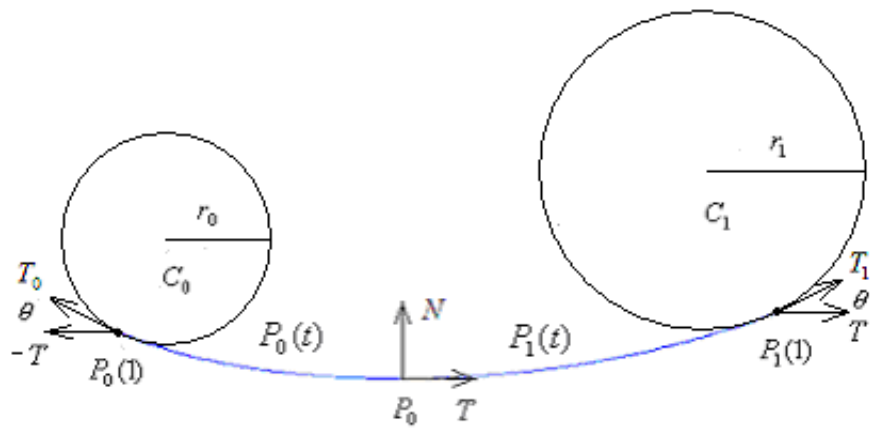

Fig. 2. G2 transition curve in $\mathrm{C}$ shape between two separated circles

In this case, assume that two circles $\Omega_{0}, \Omega_{1}$ centred at $C_{0}, C_{1}$ with radius $r_{0}, r_{1}, r_{0} \geq r_{1}$, see Fig 2 . We use a pair of quintic PH Spirals $P_{0}(t), P_{1}(t)$ to construct the transition curve. Suppose $P_{0}(0), P_{1}(0)$ are the joint of two spirals at $P_{0}$. Points $P_{0}(1), P_{1}(1)$ connect two circles, $T, N$ are the unit tangent vector and the unit normal vector at $P_{0}$, points $T_{0}, T_{1}$ are the unit tangent vector at 
another endpoints of two curves, the angle from $T$ to $T_{1}$ and the angle from $T_{0}$ to $T$ are both $\theta$.

Let $\varepsilon=\frac{6}{7}\left(\frac{\left\|C_{1}-C_{0}\right\|}{\left\|r_{1}-r_{0}\right\|}-1\right), x=\left\{\begin{array}{l}1 \text { when } x \leq 1 \\ \varepsilon \text { when } x>1\end{array}, y=\sqrt{\frac{10}{7}}\right.$, by the geometry relation, we can obtain the following cubic equation

$h(\theta)=\left\|C_{1}-C_{0}\right\|^{2}(1+\cos \theta)^{3}-\frac{\left(r_{1}+r_{0}\right)^{2}}{120^{2}}(1-\cos \theta) g_{1}^{2}(\theta)-\frac{\left(r_{1}-r_{0}\right)^{2}}{60^{2}}(1+\cos \theta) g_{2}^{2}(\theta)$, where $g_{1}(\theta)=(140 x-120) \cos \theta+490 x^{2}+140 x-120, g_{2}(\theta)=(60-70 x) \cos \theta+$ $70 x+60$.

we can guarantee that $h(0)>0, h\left(\frac{\pi}{2}\right)<0$ and $h^{\prime}(\theta)<0$ for $\theta \in\left(0, \frac{\pi}{2}\right)$. So there is the unique real root of $h(\theta)=0$ when $\theta \in\left(0, \frac{\pi}{2}\right)$. This root can be gotten by the rooting formula of cubic equation. In the computation process, we find the ratio of two radii has no restriction.

For the G2 transition curve in $\mathrm{S}$ shape between two separated circles, we can also use a pair of quintic PH spirals to construct the transition curve. It can be gotten by the rooting formula of cubic equation and the ratio of two radii has no restriction.

\section{Acknowledgements}

This paper was supported by National Natural Science Foundation of China (No.60373070 and No.60573147), Research Foundation of Microsoft China (Pro ject-2004-Image-01), Postdoctor Foundation of Shanghai (05R214129), Education Foundation of Zhejiang (20050786).

\section{References}

1. Guggenheimer H.W.: Differential geometry. New York: McGraw-Hill (1963)

2. Baass K.G.: The use of clothoid templates in highway design. Transportation Forum (1984) $1,47-52$

3. Meek D.S., Walton D.J.: The use of Cornu spirals in drawing planar curves of controlled curvature. Journal of Computational and Applied Mathematics (1989) 25, 69-78

4. Walton D.J., Meek D.S.: A planar cubic Bezier spiral. Journal of Computational and Applied Mathematics (1996) 72, 85-100

5. Walton D.J., Meek D.S.: A Pythagorean hodograph quintic spiral. Computer Aided Design (1996) 28, 943-950

6. Walton D.J., Meek D.S.: Planar G2 transition curves composed of cubic Bezier spiral segments. Journal of Computational and Applied Mathematics, (2003) 157, 453-476

7. Walton D.J., Meek D.S.: Planar G2 transition with a fair Pythagorean hodograph quintic curve. Journal of Computational and Applied Mathematics (2002) 138, 109126

8. Walton D.J., Meek D.S.: G2 curve composed of cubic and Pythagorean hodograph quintic spirals. Computer Aided Geometry Design (1998) 15, 547-566

9. Li Z., Meek D.S.: Smoothing an arc spline. Computers \& Graphics (2005) 29, 576587 